\title{
Clinical trials for the treatment of coronavirus disease 2019 (COVID-19): A rapid response to urgent need
}

\author{
Tengyue Zhang ${ }^{1}$, Yudi $\mathrm{He}^{2}$, Wenshuai $\mathrm{Xu}^{2}$, Aiping $\mathrm{Ma}^{3}$, Yanli Yang ${ }^{2}$ \& Kai-Feng $\mathrm{Xu}^{2 *}$ \\ ${ }^{1}$ Department of Internal Medicine, Peking Union Medical College Hospital, Chinese Academy of Medical Sciences, Beijing 100730, China; \\ ${ }^{2}$ Department of Pulmonary and Critical Care Medicine, Peking Union Medical College Hospital, Chinese Academy of Medical Sciences, \\ Beijing 100730, China; \\ ${ }^{3}$ Department of Pulmonary and Critical Care Medicine, The First Affiliated Hospital, College of Medicine, Xiamen University, \\ Xiamen 361003, China
}

Received February 23, 2020; accepted February 28, 2020; published online February 29, 2020 $\begin{array}{ll}\text { Citation: } & \text { Zhang, T., He, Y., Xu, W., Ma, A., Yang, Y., and Xu, K.F. (2020). Clinical trials for the treatment of coronavirus disease } 2019 \text { (COVID-19): A rapid } \\ \text { response to urgent need. Sci China Life Sci 63, 774-776. htps://doi.org/10.1007/s11427-020-1660-2 }\end{array}$

Emerging coronavirus disease 2019, known as COVID-19, is caused by the newly identified SARS-CoV-2 virus. Since the outbreak of COVID-19 in December 2019 in Wuhan (China), more than 70 thousand patients were affected. The disease resulted in 1,870 deaths by February 18, 2020, according to the National Health Commission of People's Republic of China (http://www.nhc.gov.cn). The number of patients diagnosed and death is still on the rise. Unfortunately thus far, no proven effective treatment is available for patients contracted this emerging infection. Experience in the management of coronavirus epidemic of severe acute respiratory syndrome (SARS) and the Middle East respiratory syndrome (MERS) has limited applicability for treating COVID-19. The Chinese health care teams have been fully engaged at the frontline to deal with this COVID19 epidemic. They are also actively conducting many scientific studies on the pathogenesis of the diseases caused by this virus, mode of transmission, clinical profiles, management, and disease prevention.

Clinical trial for COVID-19 was first registered on January 23,2020 . In the last few weeks, an escalating number of clinical trials has been planned and registered with ongoing investigations taking place. From the Chinese Clinical Trial Registry (http://www.chictr.org.cn) and U.S. National Li-

\footnotetext{
*Corresponding author (email: xukf@pumch.cn)
}

brary of Medicine Clinical Trial Registry (https://clinicaltrials.gov), there are 125 clinical trials registered by February 18, 2020, focusing on the treatment of COVID-19. There is an upward trend on the number of registered clinical trials (Figure 1). Among the 125 clinical trials on treatment, $33.3 \%$ used anti-viral agents, $14.7 \%$ anti-inflammation or immunomodulators, $33.3 \%$ herbs or traditional Chinese medicine (TCM), 9.3\% cell-based therapy, $2.3 \%$ antioxidation and $7.0 \%$ other approaches. Pharmaceutical companies, government, institutions, physicians and scientists are the main force behind these researches.

17 years ago (2003), SARS affected 8,096 cases with $9.6 \%$ fatality within only a few months (http://www.who.int/csr/ sars/country/table2004_04_21/en/). The China's health care system has responded quickly this time with coronavirus epidemic through active engagement by Chinese scientists in researching for the treatment and control of the spread of COVID-19. For the first time, TCM is collectively tested within a framework of modern medical research during an outbreak of infectious disease.

Among clinical trials that have been registered, anti-viral and anti-inflammatory or immunomodulation treatment are of great interest to the investigators. The first anti-viral clinical trial with Lopinavir/Ritonavir has already reached the targeted sample size of 160 cases. The data on the outcome of the treatment are currently being analyzed and re- 

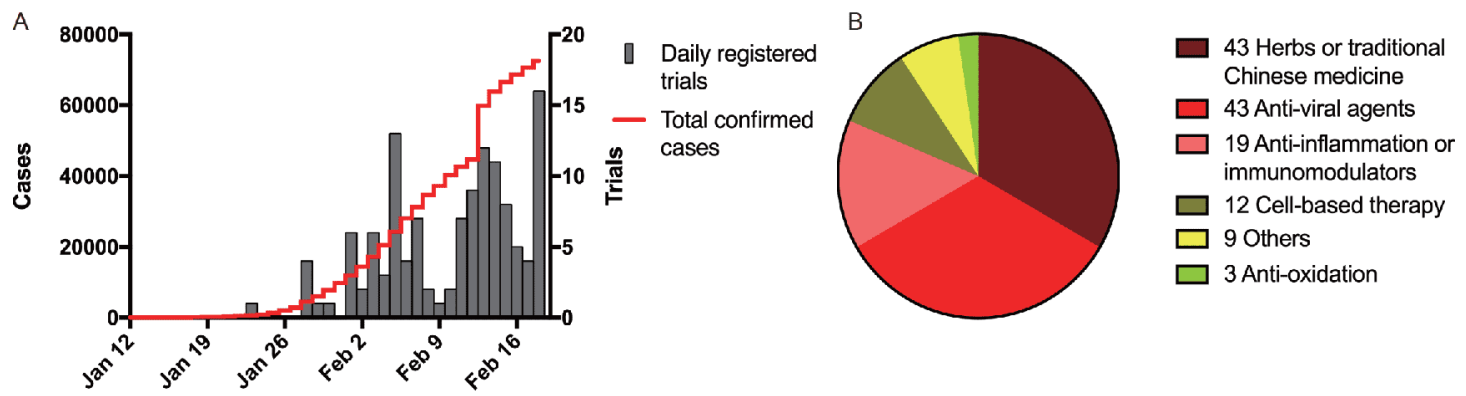

Figure 1 Rapidly growing registered clinical trials on COVID-19 in China. A, The curve represents accumulated numbers of COVID-19 cases since January 12, 2020. Daily registered clinical trials numbers are also shown. B, Classification of all 125 clinical trials until February 18, 2020. Among them, four clinical trials tested two treatments each.

sults are expected to be revealed soon. The anti-coronavirus activity of Lopinavir/Ritonavir has already been found from SARS clinical cases in the past (Chan et al., 2003). There are ongoing clinical trials on the use of Remdesivir with an ambitious targeted sample size of 760 patients. Remdesivir, an adenosine analog, when incorporated into nascent viral RNA chains, results in premature termination of viral replications. This has been found to be a promising antiviral drug against a wide array of RNA viruses including SARSCoV and MERS-CoV (Wang et al., 2020). As shown in Table $\mathrm{S} 1$ and Table S2 in supporting information, there are a large number of anti-viral agents being used in the registered clinical trials in China. Chloroquine or Hydroxychloroquine is highly expected to be a promising anti-SARS-CoV-2 activity in vitro (Wang et al., 2020). It is worth noting that this novice way of using old drugs for treating coronavirus may prove to be a quick way to find an effective treatment.

The pathogeneses of diseases caused by COVID-19 are very unclear so far, ranging from acute damage to alveolar epithelium, inflammation, cytokine storm to multiple organ failure. The presence of decreased lymphocytes and increased inflammatory mediators in affected cases suggest the likelihood of inflammatory mechanisms being involved in the pathogenesis (Huang et al., 2020). The anti-inflammatory approach was adopted by some scientists using such agents as corticosteroids, immunoglobins or targeted therapy such as anti-interleukin 6 therapy. The plasma containing antibodies subsequent developed during the convalescent phase from infected patients may prove to be valuable in treating COVID-19 patients.

Surprisingly, one-third of the registered clinical trials are given TCM. TCM medicine, usually combined with modern medicine, has being used widely in patients with COVID-19. A meta-analysis of TCM in treating SARS in the past suggested TCM was effective in relieving symptom of fever, requiring lesser dosage of corticosteroid and resulting in better improvement in CD4 count and reducing pulmonary infiltrate (Chen et al., 2007). The combination of TCM with modern medicine sounds promising for treating diseases caused by coronavirus. Other types of new treatments have been developed - such as stem cells, anti-PD-L1 monoclonal antibody, etc.

Table S1 in supporting information is not a complete list as the number of registered clinical trials is still arising. Also, some other important approaches that are not listed so far are expected to be navigated in future studies such as finding effective monoclonal antibodies or vaccines for COVID-19. During an epidemic crisis such as this coronavirus outbreak, it is an impossible mission to conduct such a huge number of clinical trials without risking the quality of research being compromised. When conducting many clinical trials during an epidemic, there are a number of pressing issues that need to be addressed.

Firstly, how to use valuable resources efficiently is a question that needs to be answered. If all 125 trials are performed as planned, a total of 19,749 subjects are required to conduct such trials. As suggested in a recent commentary in Nature, a master plan guiding the protocol and standards of research design in patient recruitment, randomization and measurements of outcomes will be required. When there is a standardized protocol, then one can pool knowledge gained from the different trials for comparison of the effectiveness of various approaches (Maxmen, 2020). However, the cost of all those 125 registered trials is enormous. Thus, every effort should be made to avoid repetition of the same clinical trials. We advocate the establishment of a national system of sharing data and keeping database for analysis in order to share knowledge for the advancement of effective treatment and to avoid wasting of national financial and human resources.

Secondly, the rationale for conducting clinical trials should be carefully examined. Currently, it is difficult to know the rationale behind some clinical trials. Before we start to write a protocol, we need to examine what the results of in vitro and in vivo studies of a potential intervention that we have known. There are other questions such as "what is the evidence on the pathogenesis in COVID-19 or other coronavirus infections? What are the preliminary results in previous studies on human?" etc.

Thirdly, ethical consideration is always a challenge in emerging infectious diseases and critical care medicine. For 
example, in the design of Remdesirvir trials (NCT04252664, NCT04257656), the placebo was used with the exclusion of any other experimental treatment. The very strict requirements of using a placebo may cause harm to the patients under study for sacrificing the potential best patient care. This is pertinent when clinical trials are undertaken among critically ill patients in ICU as there is a need to divide the cohort equally between study and control groups when placebo or other comparators is used. Potential risk of intervention investigated should be fully evaluated and minimized. Therefore, we recommend that the Institution Review Board (IRB) from different institutions should scrutinize the protocols submitted for clinical trials very carefully before approving. To date, many of the protocols posted have not yet received local IRB approval. We also recommend the establishment of a national IRB by the National Health Commission during the epidemic crisis to review effectively these ethical issues.

Fourthly, it is difficult to provide a research team specifically designated to conduct and collect data, monitoring the research process as well as examining the outcomes of clinical measurements during the outbreak of infectious diseases. Specific funding should be allocated for the establishment of this team in strict personal-entry-limited wards and hospitals. Currently we depend on individual physicians and nurses in wards without enough research support team.

Fifthly, strict rules should be applied for conducting clinical investigations to ensure high quality of the trials. Strict rules should be laid for following the study protocols carefully, such as subject selection, randomization and blinding, outcome measurement and data collection. The informed consent should be obtained formally. High standard of the study completed should be maintained. In another word, the standards of scientific investigations should never be lost even in crisis time such as the COVID-19 epidemic. Otherwise our efforts will be going nowhere.

Finally, it is hoped that any positive outcome from clinical studies will be reasonably and timely translated into quality clinical practice. The results from the studies will guide towards not only the current COVID-19 treatment but also the management of potential outbreaks of other severe viral respiratory infections in the future. Although it is particularly challenging to assess the efficiency of interventions during an infectious disease outbreak, the effort of trying is worthwhile and the knowledge gain will be very valuable. We are not sure how many clinical trials will be finally performed and completed, but we are certain that this will be the first effort to get valuable knowledge through thoroughly scrutinized investigations and well-tested outcomes about an emerging infectious disease such as COVID-19.

Compliance and ethics The author(s) declare that they have no conflict of interest.

Acknowledgements We thank Dr. Guodong Fang, a former infectious disease professor from the Peking Union Medical College Hospital and senior medical officer at the U.S. Food and Drug Administration, for helpful discussion. We thank Ms. Yu Li for her assistance in data collection. We also thank Dr. Bee Hong Lo, a visiting consultant pediatrician at the Children's Hospital at Westmead, for editing and proofreading this article.

\section{References}

Chan, K.S., Lai, S.T., Chu, C.M., Tsui, E., Tam, C.Y., Wong, M.M., Tse, M. W., Que, T.L., Peiris, J.S., Sung, J., et al. (2003). Treatment of severe acute respiratory syndrome with lopinavir/ritonavir: a multicentre retrospective matched cohort study. Hong Kong Med J 9, 399-406.

Chen, Y., Guo, J.J., Healy, D.P., and Zhan, S. (2007). Effect of integrated traditional Chinese medicine and western medicine on the treatment of severe acute respiratory syndrome: A meta-analysis. Pharmacy pract (Granada Ed impr) 5, 1-9.

Huang, C., Wang, Y., Li, X., Ren, L., Zhao, J., Hu, Y., Zhang, L., Fan, G., $\mathrm{Xu}, \mathrm{J}$., Gu, X., et al. (2020). Clinical features of patients infected with 2019 novel coronavirus in Wuhan, China. Lancet 395, 497-506.

Maxmen, A. (2020). More than 80 clinical trials launch to test coronavirus treatments. Nature 578, 347-348.

Wang, M., Cao, R., Zhang, L., Yang, X., Liu, J., Xu, M., Shi, Z., Hu, Z., Zhong, W., and Xiao, G. (2020). Remdesivir and chloroquine effectively inhibit the recently emerged novel coronavirus (2019$\mathrm{nCoV})$ in vitro. Cell Res doi: 10.1038/S41422-020-0282-0.

\section{SUPPORTING INFORMATION}

Table S1 Therapeutic approaches in registered clinical trials on coronavirus disease 2019 (COVID-19)

Table S2 Basic information of 125 clinical trials

The supporting information is available online at http://life.scichina.com and https://link.springer.com. The supporting materials are published as submitted, without typesetting or editing. The responsibility for scientific accuracy and content remains entirely with the authors. 